\title{
A prospective study of clinical profile of patients with acute kidney injury following acute gastroenteritis
}

\author{
Dnyaneshwar Malharrao Ghuge ${ }^{1 *}$, Balasaheb Eknathrao Karad ${ }^{2}$
}

${ }^{1}$ Associate Professor, ${ }^{2}$ Professor, Department of Medicine, Pacific Institute of Medical Sciences, Udaipur, Rajasthan, INDIA. Email: drdmghuge@yahoo.com, karadbalasaheb@gmail.com

\begin{abstract}
Background: Increasing number of patients of AGE are now diagnosed with AKI.. Over the recent years there has been increasing recognition that relatively small rises in serum creatinine in a variety of clinical settings are associated with worse outcomes. In present study, we aimed to study clinical profile of patients with acute kidney injury following acute gastroenteritis at our tertiary hospital. Material and Methods: Present study was conducted in patients who were diagnosed to have AKI following acute gastroenteritis. Statistical analysis was done using descriptive statistics. Results were expressed as mean and standard deviation for continuous data and frequency as number and percentage. Results: After applying inclusion and exclusion criteria, total 72 patients of AGE with AKI were considered for this study. Most common age group in this study was age group of 61-70 years (28\%), followed by age group of 51-60 years (25\%). Mean age of study patients was $53.8 \pm 11.7$ years. Male patients $(64 \%)$ were more than female patients $(36 \%)$. Male to female ratio was 1.8:1. According to clinical presentation most common symptom was loose stools $(100 \%)$, followed by fever $(76 \%)$ and vomiting $(68 \%)$. Other complaints were shortness of breath $(18 \%)$ and altered sensorium $(8 \%)$. Diabetes was the most common co-morbidity noted (39\%) followed by hypertension (34\%). AKI was staged for severity according to the KDIGO criteria. At the time of diagnosis most patients were in stage $1(57 \%)$, while $32 \%$ and $11 \%$ were in stage 2 and 3 respectively. In this study hypotension, hyperkalemia were common complications of Acute Kidney Injury. Other complications were metabolic acidosis, encephalopathy, pulmonary edema, anemia, multi organ dysfunction syndrome (MODS), hypokalemia and hyponatremia. In present study, $11 \%$ patients underwent hemodialysis and $3 \%$ mortality was noted. Conclusion: Acute kidney injury in patients with acute gastroenteritis had good prognosis if detected earlier. Early recognition of AKI is essential to ensure prompt and appropriate management, and to avoid progression to deadlier stages of the disease.

Keywords: Acute Gastroenteritis, Acute Kidney Injury, KDIGO criteria
\end{abstract}

\section{*Address for Correspondence:}

Dr Dnyaneshwar Malharrao Ghuge, Associate Professor, Department of Medicine, Pacific Institute of Medical Sciences, Udaipur, Rajasthan, INDIA.

Email: karadbalasaheb@gmail.com

Received Date: 20/12/2019 Revised Date: 11/01/2020 Accepted Date: 29/01/2020

DOI: https://doi.org/10.26611/102116112

This work is licensed under a Creative Commons Attribution-NonCommercial 4.0 International License. $(\mathrm{cc})$ ) EY-NC

\begin{tabular}{|l|l|}
\hline \multicolumn{2}{|c|}{ Access this article online } \\
\hline Quick Response Code: & Website: \\
\hline & www.medpulse.in \\
\hline
\end{tabular}

\section{INTRODUCTION}

Acute kidney injury (AKI) previously known as Acute Renal Failure, is a clinical syndrome characterized by an abrupt decline in glomerular filtration rate sufficient to decrease the elimination of nitrogenous waste products (urea and creatinine) and other uremic toxins. ${ }^{1}$ The causes of AKI have traditionally been divided into three broad categories as prerenal (due to renal hypoperfusion), intrinsic (due to renal parenchymal pathology), postrenal (due to urinary tract obstruction). ${ }^{2}$ Common causes for AKI are acute volume depletion (diarrheal diseases, haemorrhage), sepsis, infection (malaria, pneumonia, viral hepatitis), snake bite, acute cardiac failure, nephrotoxic 
drug use, malignancy, SLE, hypertension, major surgeries, radio contrast agents. etc. ${ }^{3}$ Severe acute gastroenteritis (AGE) may lead to acute fluid loss followed by oliguria, azotemia, and hyperkalemia (due to supervening catabolic state and lactic acidosis) renal hypoperfusion and AKI. This may progress to renal shutdown with oligoanuria and its functional consequences with or without significant structural damage. Increasing number of patients of AGE are now diagnosed with AKI.. Over the recent years there has been increasing recognition that relatively small rises in serum creatinine in a variety of clinical settings are associated with worse outcomes. ${ }^{4}$ In present study, we aimed to study clinical profile of patients with acute kidney injury following acute gastroenteritis at our tertiary hospital.

\section{MATERIAL AND METHODS}

Present study was conducted in patients who were diagnosed to have AKI following acute gastroenteritis. Study was prospective, observational type. Study was conducted in Department of General Medicine, Pacific Institute of Medical Sciences, Udaipur, between October 2018 to October 2019. Permission to conduct present study was granted by ethical committee.

Inclusion Criteria

- Patients above 18 years, admitted with acute gastroenteritis.

- Patients with acute kidney injury (AKI), diagnosed by KDIGO criteria

Exclusion Criteria

- Age less than 18 years

- Patients with chronic renal insufficiency

- Patients who are initially considered as AKI but subsequently found to be suffering from longstanding renal disease.

An informed consent was taken from patients for participation. Demographic and clinical details, thorough examination findings were noted. Renal function tests with electrolytes are done daily and recorded. Other laboratory parameters such as CBC, ESR, Urine examination, Stool Examination, HIV, blood glucose, liver function test are also done. ABG, ECG, abdominal ultrasound examination were done whenever required. AKI was diagnosed according to Acute Kidney Injury Working Group of KDIGO (Kidney Disease: Improving Global Outcomes) ${ }^{2}$ which diagnoses AKI by an absolute increase in Serum Creatinine, at least $0.3 \mathrm{mg} / \mathrm{dL}$ within 48 hours or by a $50 \%$ increase in Serum Creatinine from baseline within 7 days, or a urine volume of less than $0.5 \mathrm{~mL} / \mathrm{kg} / \mathrm{h}$ for at least 6 hours. Primary treatment was done with adequate fluid replacement and antibiotics (gram negative spectrum for ongoing gastroenteritis). Dialysis was done in patients with hyperkalemia, pulmonary edema and severe metabolic acidosis, who did not respond to medical treatment and prophylactically in patients whose creatinine is more than $4 \mathrm{mg} / \mathrm{dl}$. Data recorded include patients' characteristics, comorbid medical conditions, dialysis requirement, total duration of hospital stay, and complications that occurred in their hospital stay were recorded. and final outcome. The clinical and laboratory parameters were analyzed to assess the role of each of these factors as the possible outcome i.e. recovery or death. All patients were followed up till discharge or death. Statistical analysis was done using descriptive statistics. Results were expressed as mean and standard deviation for continuous data and frequency as number and percentage.

\section{RESULTS}

After applying inclusion and exclusion criteria, total 72 patients of AGE with AKI were considered for this study. Most common age group in this study was age group of $61-70$ years $(28 \%)$, followed by age group of 51-60 years $(25 \%)$. Mean age of study patients was $53.8 \pm 11.7$ years. Male patients $(64 \%)$ were more than female patients $(36 \%)$. Male to female ratio was 1.8:1.

Table 1: Age distribution

\begin{tabular}{cccc}
\hline Age groups (years) & Male & Female & Total \\
\hline $19-30$ & $1(1 \%)$ & $1(1 \%)$ & $2(3 \%)$ \\
$31-40$ & $3(4 \%)$ & $4(6 \%)$ & $7(10 \%)$ \\
$41-50$ & $8(11 \%)$ & $5(7 \%)$ & $13(18 \%)$ \\
$51-60$ & $12(17 \%)$ & $6(8 \%)$ & $18(25 \%)$ \\
$61-70$ & $13(18 \%)$ & $7(10 \%)$ & $20(28 \%)$ \\
$71-80$ & $7(10 \%)$ & $2(3 \%)$ & $9(13 \%)$ \\
$>80$ & $2(3 \%)$ & $1(1 \%)$ & $3(4 \%)$ \\
\hline Total & $46(64 \%)$ & $26(36 \%)$ & 72 \\
\hline
\end{tabular}

According to clinical presentation most common symptom was loose stools (100\%), followed by fever (76\%) and vomiting $(68 \%)$. Other complaints were shortness of breath $(18 \%)$ and altered sensorium (8\%). Diabetes was the most common co-morbidity noted (39\%) followed by hypertension (34\%).

Table 2: Symptom wise distribution

\begin{tabular}{ccc}
\hline Number of cases & Number of patients & Percentages \\
\hline Loose stools & 72 & $100 \%$ \\
Fever & 55 & $76 \%$ \\
Vomiting & 49 & $68 \%$ \\
Shortness of breath & 13 & $18 \%$ \\
Altered sensorium & 11 & $15 \%$ \\
\hline
\end{tabular}

AKI was staged for severity according to the KDIGO criteria $^{2}$. At the time of diagnosis most patients were in stage $1(57 \%)$, while $32 \%$ and $11 \%$ were in stage 2 and 3 respectively. 
Table 3: AKI staging (KDIGO)

\begin{tabular}{cccc}
\hline Stage & Serum creatinine & Urine output & No. of patients (\%) \\
\hline 1 & $1.5-1.9$ times baseline OR & $<0.5 \mathrm{ml} / \mathrm{kg} / \mathrm{h}$ for $6-12$ hours & $41(57 \%)$ \\
2 & $\geq 0.3 \mathrm{mg} / \mathrm{dl}(\geq 26.5 \mu \mathrm{mol} / \mathrm{l})$ increase & & $23(32 \%)$ \\
3 & $2.0-2.9$ times baseline & $<0.5 \mathrm{ml} / \mathrm{kg} / \mathrm{h}$ for $\geq 12$ hours & $<0.3 \mathrm{ml} / \mathrm{kg} / \mathrm{h}$ for $\geq 24$ hours \\
& 3.0 times baseline OR & OR & Anuria for $\geq 12$ hours \\
& Increase in serum creatinine to $\geq 4.0 \mathrm{mg} / \mathrm{dl}(\geq 353.6 \mu \mathrm{mol} / \mathrm{l})$ OR & & $8(11 \%)$ \\
& Initiation of renal replacement therapy OR & &
\end{tabular}

In this study hypotension, hyperkalemia were common complications of Acute Kidney Injury. Other complications were metabolic acidosis, encephalopathy, pulmonary edema, anemia, multi organ dysfunction syndrome (MODS), hypokalemia and hyponatremia. In present study, $11 \%$ patients underwent hemodialysis and $3 \%$ mortality was noted.

\section{DISCUSSION}

In developing countries AGE still remains one of the common causes of communicable disease that lead to AKI. ${ }^{5}$ Non-availability of clean drinking water, adulteration of food and beverages, poor sanitation, lack of public awareness about personal hygiene are common causes of AGE in developing countries. Most patients with mild to moderate acute kidney injury are asymptomatic and are identified on laboratory testing. Patients with severe cases of acute kidney injury, however, may be symptomatic and present with listlessness, confusion, fatigue, anorexia, nausea, vomiting, weight gain, or edema. ${ }^{6}$ Lewington AJ noted that the pooled incidence rates of AKI in adult was $21.6-20 \%$ of hospitalized adult patients experienced AKI during a hospital care which is associated with high expenditure of resources and lead to adverse outcomes. ${ }^{7}$ AKI with a rise in serum creatinine as modest as $0.3 \mathrm{mg} / \mathrm{dL}$ is associated with a $70 \%$ increase in mortality risk and increased risk of death by 6.5 times. $^{8}$ Early in the course of AKI, optimization of the hemodynamic status and correction of any volume deficit will have a salutary effect on kidney function. Diuretics do not have any significant effect on progression or outcome of AKI. ${ }^{9}$ In a similar study, mean age of presentation 46.5 \pm 11.35 years, in males was $47.3 \pm 11.35$ and that of females was $45.72 \pm 10.23$ years. $63.33 \%$ were Males and $36.7 \%$ were females. Similar findings were noted in present study. In the study by Satri V et al. sepsis was found to be the most common cause of AKI followed by acute gastroenteritis, while Eswarappa $\mathrm{M}$ et al. noted acute gastroenteritis as most common cause of AKI in their study. On stool culture and sensitivity, we found $88.5 \%$ patients with sterile stool cultures. $10.3 \%$ patients had E. coli on culture while 1 patient has Salmonella growth on stool culture. ${ }^{13}$ Similar findings were noted in present study. Pajai AE et al.., ${ }^{14}$ noted that vasopressor support was required in $18.9 \%$ patients, $7.5 \%$ required transfusion of blood and blood products, $11.3 \%$ required CPAP support and 2 patients needed mechanical ventilation. $50.9 \%$ recovered within 7 days of treatment initiation and $45.3 \%$ required more than 7 days for recovery. $32.1 \%$ required renal replacement therapy and $3.8 \%$ mortality was noted. Analysis revealed delay in initiation of definitive therapy lead to increased requirement of supportive therapy in form of Vasopressor support, renal replacement therapy and it was statistically significant. ${ }^{15}$ Inbanathan $\mathrm{J}$ et al.., ${ }^{15}$ noted that $70 \%$ patients were treated conservatively and $30 \%$ required hemodialysis. $52 \%$ were discharged within 1 week, while $37 \%$ of patients were discharged between 1-3 weeks. $11 \%$ of patients were treated for more than 3 weeks. $4 \%$ mortality was noted. The prognosis of patients with AKI is directly related to cause of renal failure and, to great extent, to the duration of renal failure before therapeutic intervention. Prognostic factors are older age, multiorgan failure (i.e., the more the organ that fails, the worser is prognosis), circulatory failure, vasopressor support, and need for renal replacement therapy (RRT). Factors influencing patient survival in acute kidney injury are severity of injury and underlying disease and other factors such as age, severity of coexistent illnesses and associated complications like Intravascular overload, hyperkalemia and other metabolic complications and systemic life-threatening complications like cardiac arrhythmia, myocardial infarction, pulmonary embolism, gastrointestinal ulcers, seizures, coma, hemolysis, bleeding tendencies and severe infections. ${ }^{16}$ Regardless of cause, the management of AKI is mainly supportive, with dialysis being indicated when medical management fails to treat the complications. The duration and severity of AKI is a risk factor for the development of complications such as a 10-fold increase in the risk of chronic kidney disease and a 3-fold risk of end-stage renal disease. ${ }^{17,18}$ Failure to detect and treat timely and/or adequately usually leads to significant untoward consequences. It is associated with a high morbidity and permanent loss of kidney function. ${ }^{19}$ In the appropriate context, early detection requires a high degree of suspicion that AKI is occurring. Diagnosis requires a combination of a clinical history, a thorough physical examination, an accurate assessment of kidney function, appropriate imaging, and when indicated, a kidney biopsy. 


\section{CONCLUSION}

Acute kidney injury in patients with acute gastroenteritis had good prognosis if detected earlier. Early recognition of AKI is essential to ensure prompt and appropriate management, and to avoid progression to deadlier stages of the disease.

\section{REFERENCES}

1. Susantitaphong P, Cruz DN, Cerda J, Abulfaraj M, Alqahtani F, Koulouridis I, et al.. World incidence of AKI: A meta-analysis. Clin J Am Soc Nephrol2013; 8: 1482-93.

2. Kidney Disease: Improving Global Outcomes (KDIGO) Acute Kidney Injury Work Group. KDIGO clinical practice guideline for acute kidney injury. Kidney Int., 2012; 2(Suppl 1): 1-138.

3. Mehta RL, Kellum JA, Shah SV, et al.. Acute kidney injury network: report of an initiative to improve outcomes in acute kidney injury. Crit Care. 2007;11: R31.

4. Praught ML, Shlipak MG. Are small changes in serum creatinine an important risk factor? Curr Opin Nephrol Hypertens 2005; 14:265-70.

5. Khakurel S, Satyal PR, Agrawal RK, Chhetri PK, Hada R. Acute Renal Failure in tertiary care centre in Nepal. J Nepal Med Assoc. 2005 Apr-Jun;44(158):32-5.

6. Meyer TW, Hostetter TH. Uremia. N Engl J Med., 2007; 357(13): 1316-1325.

7. Lewington AJ, Cerdá J, Mehta RL. Raising awareness of acute kidney injury: A global perspective of a silent killer. Kidney Int 2013;84:457-67.

8. Soren B, Papareddy A, Kommareddy SR, Meriga RK, Midathala NV, Sarikonda GR. Clinical profile of acute kidney injury in patients admitted to medical wards in a tertiary care setting. Int $\mathrm{J}$ Med Sci Public Health 2019;8(2):110-113.

9. Ho KM, Sheridan DJ: Meta-analysis of frusemide to prevent or treat acute renal failure. BMJ 333: 420, 2006.
10. Dr. G. Swarnalatha Devi, Study on clinical profile and outcome of acute kidney injury in acute gastroenteritis in a tertiary care centre, Indian journal of applied research, volume-8, Issue-11, November-2018

11. Satri V, Kumar VS, Satyanarayana V, Ramakrishna BS, Srinivasa Rao PVLN, Madhusudan M. Epidemiology and outcome of acute kidney injury in patients presenting to emergency department - Our experience. J Clin Sci Res 2020;9:77-81.

12. Eswarappa M, Gireesh MS, Ravi V, Kumar D, Dev G. Spectrum of acute kidney injury in critically ill patients: A single center study from South India. Indian J Nephrol 2014;24:280-5.

13. Paritosh Desai,Vasant Deokar. Study of acute renal failure due to gastroenteritis in a tertiary health care centre. JCR. 2020; 7(12): 380-383.

14. Pajai AE, Mehta KS, Bhurke SP, Shirkande AK, Pagar S, Pradhan RU. Observational study to assess demographic, clinical profile and outcome of acute kidney injury due to acute gastroenteritis during monsoon season. Int $\mathrm{J}$ Adv Med 2019;6:xxx-xx.

15. Inbanathan J, Lavanya BU. Clinical Profile of Renal Involvement in Acute Gastroenteritis Patients. Int J Sci Stud 2016;4(8):48-52.

16. Sean M. Bagshaw .Short- and long-term survival after acute kidney injury Nephrol. Dial. Transplant. 2008;23:23-29

17. Chawla LS, Amdur RL, Amodeo S, Kimmel PL, Palant CE. The severity of acute kidney injury predicts progression to chronic kidney disease. Kidney Int 2011;79:1361-9.

18. Coca SG, Singanamala S, Parikh CR. Chronic kidney disease after acute kidney injury: A systematic review and meta-analysis. Kidney Int 2012;81:442-8.

19. Wald R, Quinn RR, Luo J, Li P, Scales DC, Mamdani MM, et al.. Chronic dialysis and death among survivors of acute kidney injury requiring dialysis. JAMA 2009;302:1179-85.

\section{Source of Support: None Declared Conflict of Interest: None Declared}

Policy for Articles with Open Access:

Authors who publish with MedPulse International Journal of Medicine, Print ISSN: 2550-7583, Online ISSN: 2636-4751 agree to the following terms: Authors retain copyright and grant the journal right of first publication with the work simultaneously licensed under a Creative Commons Attribution License that allows others to share the work with an acknowledgement of the work's authorship and initial publication in this journal.

Authors are permitted and encouraged to post links to their work online (e.g., in institutional repositories or on their website) prior to and during the submission process, as it can lead to productive exchanges, as well as earlier and greater citation of published work. 